\title{
Casual blood pressure and ambulatory blood pressure measurement in children
}

\author{
Pediatric Nephrology Unit, Instituto da Criança, Hospital das Clínicas, \\ Universidade de São Paulo, São Paulo, Brazil.
}

Cardiovascular diseases are the main causes of death in Brazil. Stroke mortality rates among Brazilians are high, reflecting the burden of hypertension. Some international epidemiological studies on blood pressure among children and adolescents have revealed that blood pressure levels in childhood are the strongest predictor of adult blood pressure levels. ${ }^{1-3}$ In the adult population, hypertension causes a two to threefold increase in an individual's risk of cardiovascular morbidity. ${ }^{4,5}$ The relationship between hypertension and cardiovascular disease seems to be continuous: cardiovascular risk depends on blood pressure itself, coexistent risk factors and whether there is hypertensive end-organ damage.

As accuracy in determining blood pressure is essential, a standardized protocol should be considered for blood pressure measurement, which would make the comparison of results obtained by different studies in different countries possible. Observers should be trained and certified to minimize measurement bias. Homogeneous decisions should be taken regarding equipment factors such as an appropriate cuff bladder size or the alternative use of mercury manometers or oscillometric devices. Technical factors such as the recording of fourth, fifth or both Korotkoff sounds for diastolic blood pressure need to be taken into consideration. Also, the number of measurements needed for estimating a child's blood pressure and the influence on its measured value of environmental factors such as the time of the day and ambient temperature must be considered. ${ }^{6}$ Some of these factors will be discussed separately in the next paragraphs.
The cuff

Classically, to obtain an accurate blood pressure measurement, a cuff bladder width of approximately $40 \%$ of the upper arm circumference should be chosen because it most closely approximates intra-arterial readings. ${ }^{7}$ The bladder length should be at least $90 \%$ of arm circumference to avoid overestimation of blood pressure, especially in children. ${ }^{8}$ Another less-known effect of the cuff size change occurs when, in accordance with the abovementioned instructions for cuff selection, the cuff size is changed to a larger one. In this case, the cuff change leads to an abrupt fall in the value of measured blood pressure that is not arm-dependent, but cuff-dependent. ${ }^{9}$ This very inconvenient effect may be responsible for two issues: 1. Any association between blood pressure and arm circumference, such as body mass, will be biased towards zero. 2. In longitudinal studies, when changing to a larger cuff, measured blood pressure is lower than previous readings, which could lead to inappropriate inverse correlations of blood pressure with chronological age or height. In 1999, Arafat and Mattoo ${ }^{10}$ reviewed commercially available blood pressure cuffs and detected that the sizes of available cuffs, labeled as infant, pediatric, small adult, adult and large adult were heterogeneous among the different manufacturers. These authors concluded that cuff sizes need to be standardized and indicate bladder size, and suggested that they should be color-coded for convenience.

Number of measurements needed

Another important issue to consider is the number of measurements that should be re-

Some epidemiological studies on blood pressure among children and adolescents have revealed that blood pressure levels in childhood are the strongest predictors of adult blood presvere sure levels. In the adult population, hypertension causes a two to threefold increase in an individual's risk of cardiovascular morbidity. Cardiovascular risk depends on blood pressure itself, coexistent risk factors and whether there is hypertensive end-organ damage. Therefore, hypertensine sential and a standardized protocol should be considered for blood pressure measurement, which would make the comparison of results obtained by different studies in different countries possible. This article reviews the main deerminants of accuracy for casual and ambulatory blood pressure measurements in children.

KEY WORDS: Blood. Pressure. Methodology. Children. Adolescent. 
peated within a visit and between visits in order to determine a child's blood pressure. The work by Gillman and Cook $(1993)^{6}$ demonstrated that it depends on the instrument and technique. For auscultatory equipment, using a mercury manometer or random zero manometer, among 162 children aged 8 to 12 years, the systolic blood pressure values obtained after four weekly visits with three measurements per visit leveled off after about 2-3 measurements per visit, but the difference between visits was large until about the third or fourth visit. For oscillometric equipment, using the Dinamap model 845XT, among 106 children aged 9 to 13 years, the systolic blood pressure values obtained after three weekly visits with four measurements per visit demonstrated that for the Dinamap device the first of several measurements during one particular visit was generally higher than the following ones. The values obtained started to level off after 4-5 measurements within a visit, with the "first measurement effect" reproducible even after 3 consecutive visits.

\section{The diastolic dilemma}

There has been an ongoing controversy over whether the muffling (Korotkoff 4K4) or disappearance of sounds (Korotkoff 5- K5) should be preferentially considered for the measurement of diastolic blood pressure in children. ${ }^{11}$ Neither value correctly defines intra-arterial diastolic blood pressure, since $\mathrm{K} 5$ is approximately $9 \mathrm{mmHg}$ higher than direct diastolic blood pressure and $\mathrm{K} 5$ is easier for the human ear to discern than $\mathrm{K}^{12}{ }^{12}$. Current recommendations therefore favor the use of $\mathrm{K} 5$.

The stethoscope diaphragm versus the bell

The bell is preferred for blood pressure auscultation in adults. This issue is still controversial in children, since placing the bell adequately in small children may compress the artery and produce falsely low diastolic values. Thus, some authors advocate the use of the diaphragm for small children, ${ }^{13}$ while others suggest that the bell, when properly used, should accomplish better auscultatory results. ${ }^{11}$

Time of the day and ambient temperature

It is clear from ambulatory blood pressure studies that blood pressure varies over the 24 hours of the day, presenting lower values during sleep and higher values during wakefulness, with a peak in the morning and another in late afternoon. ${ }^{14}$ There is a negative relationship between blood pressure and temperature. An increase of $10^{\circ} \mathrm{C}$ leads to a fall of approximately $5-7 \mathrm{mmHg}$ in systolic and in diastolic blood pressure. ${ }^{15,16}$

Do we have normative blood pressure data for children?

Unfortunately we don't have normative blood pressure data for the pediatric population. Table 1 shows the lack of homogeneous methodology in nine studies that made up the Second Task Force of Blood Pressure Measurement in Children, reviewed by Rosner et al. in 1993. ${ }^{17,18}$ The Update of the Second Task Force of Blood Pressure Measurement in Children added a tenth study to this list (National Health and Nutrition Examination Survey - NHANES III). ${ }^{19}$ Table 2 shows the same lack of methodological homogeneity in the six studies from which the European pediatric blood pressure normative data is at present derived..$^{20}$

It is important to emphasize that this lack of homogeneity is not a consequence of carelessness but rather of the multiple difficulties involved in performing epidemiological studies in the pediatric age group. Unfortunately, according to Nielsen et al. (1989) ${ }^{21}$, "confusion concerning the most suitable cuff... is responsible for at least some of the scatter between blood pressure studies". Arafat and Mattoo (1999), ${ }^{10}$ referring to the Update of the Second Task Force of Blood Pressure Measurement in Children, suggested that "a new multicenter study, using uniform criteria for cuff selection, may be necessary to establish the accuracy of the published nomogram on normal blood pressure in children".

What blood pressure measuring device should be used in the future?

The mercury manometer is our old friend. It is simple, accurate and easy to service. Standard $\mathrm{Hg}$ readings are the main basis for blood pressure-disease associations and, although

Table 1. Methodology parameters of the nine studies that made up the Second Task Force of Blood Pressure Measurement in Children ${ }^{17,18}$

\begin{tabular}{|c|c|c|c|c|c|c|}
\hline Source & Age (years) & Instrument & Cuff width & Cuff length & Number of observers & Place of measurement \\
\hline $\mathrm{NIH}$ & $6-17$ & Mercury column & $9.5 \times 13$ & - & Multiple - physicians & Vans \\
\hline Pittsburgh & $1-5$ & Doppler & - & $\geq 75 \% \mathrm{AC}$ & - & Home \\
\hline Dallas & $13-17$ & Random zero & Multiple AC & Most of AC & Multiple & School \\
\hline Bogalusa & $1-17$ & Mercury column & 4 cuffs AC & $\geq 50 \% \mathrm{AC}$ & 3 & School \\
\hline Houston & $3-17$ & Mercury column & $2 / 3$ arm length & $\geq 75 \% \mathrm{AC}$ & Multiple & Clinic \\
\hline South Carolina & $4-17$ & Mercury column & Multiple AC & - & Multiple & School \\
\hline lowa & $5-17$ & Doppler Random zero & 4 cuffs & - & Multiple & School \\
\hline Providence & $1-3$ & Random zero & $2 / 3$ arm length & - & Multiple & Clinic \\
\hline Minnesota & $9-17$ & Mercury column & 5 cuffs AC & $\geq 90 \% \mathrm{AC}$ & 4 & School \\
\hline
\end{tabular}

NIH: National Institutes of Health; AC: arm circumference.

Table 2. Methodology parameters for the six studies that made up the European pediatric blood pressure normative data ${ }^{19}$

\begin{tabular}{|c|c|c|c|c|c|}
\hline Source & Age (years) & Instrument & Cuff $(\mathrm{cm})$ & Position & Place of measurement \\
\hline Berlin-Bremen & $11-17$ & Random zero & $9 \times 1812 \times 2314 \times 28$ & Sitting, Right arm & school \\
\hline Cologne & $15-19$ & LSH & $12.5 \times 28$ & Sitting, Right arm & school \\
\hline Copenhagen & $6-18$ & Random zero & $6 \times 209 \times 2812 \times 35$ & Sitting, Right arm & school \\
\hline Essen & $4-18$ & LSH & $2 / 3$ arm length $8 \times 2010 \times 25$ & Sitting, Right arm & school/open \\
\hline Nancy & $4-17$ & Mercurycolumn & $2 / 3$ arm length9 $\times 2212 \times 26$ & Supine, Left arm & open \\
\hline Zoetermeer & $5-19$ & Random zero & $10 \times 2314 \times 23$ & Sitting, Left arm & open \\
\hline
\end{tabular}

LSH: London School of Hygiene equipment. 
blood pressure readings with this instrument are subject to terminal digit preference and observer bias, observer training could possibly eliminate this problem. Unfortunately, mercury has toxic effects on the environment and the mercury manometer will have to be gradually replaced.

The aneroid sphygmomanometer registers blood pressure through a mechanically intricate system. Its accuracy is affected by everyday use. When calibrated against a mercury manometer a mean difference of $3 \mathrm{mmHg}$ is acceptable, although up to $30 \%$ have errors of more than $7 \mathrm{mmHg}$. Readings are also subject to terminal digit preference and observer bias ${ }^{22}$.

What about automated sphygmomanometry? The most widely used oscillometric devices are manufactured under the name "Dinamap". Several models have been developed, each with an updated algorithm. Validation data has to be obtained separately for each model. Systolic and diastolic blood pressures are calculated as a function of the mean arterial pressure, which is the point of maximal oscillation and are calibrated to be equivalent to intra-aortic pressures. The devices are easy to use and strongly correlated to intraarterial readings. Accuracy is affected by arm movement and measurements are affected by the "first-reading effect". ${ }^{23}$

Automated oscillometric devices have to be validated before they can be recommended for clinical use. Validation protocols based on comparative measurements between oscillometric equipment and the mercury manometer were devised by the British Hypertension Society and the American Association of Medical Instruments. ${ }^{24}$ The two protocols have now been reconciled and are used in association to validate oscillometric devices. Table 3 presents the instruments currently validated and recommended for hospital use and self-measurement (home blood pressure)..$^{25,26}$

Is it possible to use auscultatory and oscillometric devices interchangeably? Unfortunately not, as Korotkoff is approximately 3 $\mathrm{mmHg}$ lower than direct systolic blood pressure and, as we mentioned earlier, K5 is approximately $9 \mathrm{mmHg}$ higher than direct diastolic blood pressure ${ }^{12}$. Park et al. $(2001)^{27}$ tested the Dinamap 8100 against the standard mercury manometer and found that the equipment detected mean systolic and diastolic blood pressure values significantly above auscultatory readings. On the other hand, Barker et al. (2000) ${ }^{28}$ tested the Omron M1 against the standard mercury manometer and concluded that the Omron M1 overestimates higher pressures and underestimates lower pressures. There is a lack of validated and approved automated devices for use in clinical and epidemiological setting for the pediatric age group. ${ }^{29}$

\section{Ambulatory blood pressure \\ monitoring in children}

The current general indications for ambulatory blood pressure monitoring are: identification of white coat hypertension, borderline hypertension, identification of nocturnal hypertension, drug resistant hypertension, indication of antihypertensive medication, hypertension of pregnancy and identification of hypotension. ${ }^{30}$ Among the current issues for ambulatory blood pressure monitoring use in pediatrics, the main problem is the lack of definite normative data. The methodology is promising, since recordings show good accuracy and reproducibility in children. ${ }^{31}$ Up-to-date definitions of sleep/wake periods, using actigraphy or a detailed diary of daily activities, are necessary for accurately determining the sleep blood pressure decline. ${ }^{32}$ The white coat effect (white coat hypertension or white coat normotension) known within the literature relating to adults has also been confirmed in the pediatric population. In the same way as for adults, the left ventricular mass index and left ventricular hypertrophy are more closely related in children to 24-hour systolic blood pressure than with casual systolic blood pressure. ${ }^{33}$ According to Kapuku et al. (1999), ${ }^{34}$ left ventricular

\section{Table 3. Automated oscillometric blood pressure measuring devices recommended for hospital use and self measurement (upper arm) ${ }^{2.3}$}

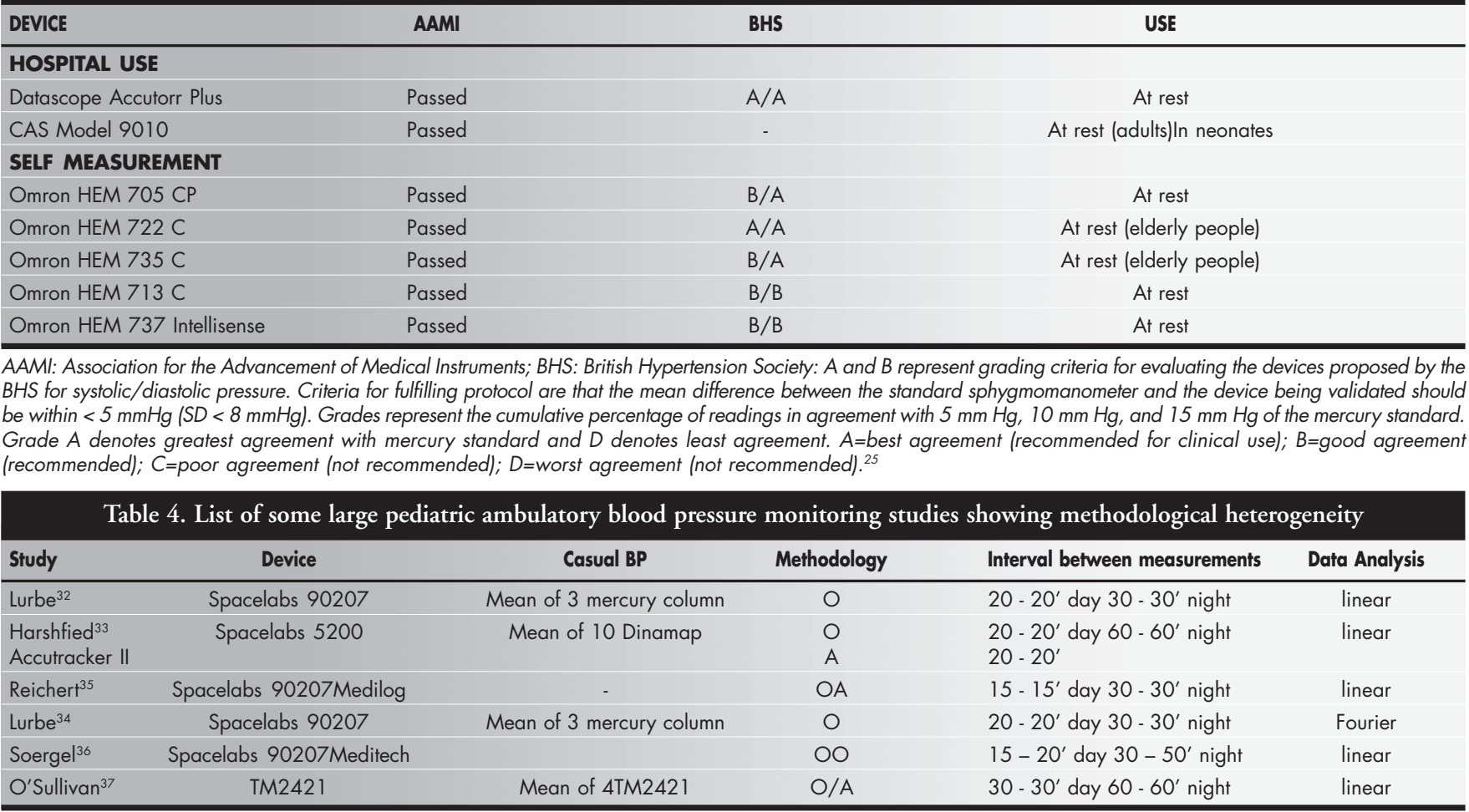

$A=$ auscultatory ; $O=$ oscillometric. 
hypertrophy can be predicted by initial ambulatory systolic parameters.

In a recent study, ${ }^{35}$ our group compared casual blood pressure and ambulatory blood pressure monitoring parameters among normotensive and hypertensive adolescents. Casual blood pressure was measured by two trained observers in two different and separate environments (clinic and ambulatory blood pressure monitoring unit). For systolic and diastolic blood pressure, in both normotensive and hypertensive populations, an alarm reaction was demonstrated during exposure to an unknown environment and observer (the ambulatory blood pressure monitoring unit). It should also be noted that, contrary to findings in adult populations, the mean casual systolic/ diastolic blood pressure measured in the clinic was lower than the mean ambulatory blood pressure monitoring parameters while awake, for normotensive and hypertensive adolescents. The same study compared findings from casual auscultatory measurements (in the clinic and ambulatory blood pressure monitoring unit) and ambulatory blood pres- sure monitoring parameters among hypertensive adolescents. The parameters included systolic and diastolic ambulatory blood pressure monitoring methods, systolic and diastolic blood pressure descent during sleep (systolic/diastolic sleep blood pressure descent), systolic and diastolic blood pressure load. This led us to conclude that, although normality parameters are still under development for ambulatory blood pressure monitoring in the pediatric age range, ambulatory blood pressure monitoring is a promising tool for the follow-up of pediatric hypertensive patients. In this respect it seems superior to casual blood pressure evaluation, since it uncovers the white coat effect.

Ambulatory blood pressure monitoring device validation data for children is scarce. The Spacelabs 90207, widely used in pediatric studies, and the TM 2421, used in a recent large pediatric study ${ }^{36}$ are equipment that has not scored well enough to be recommended according to the protocols of the British $\mathrm{Hy}$ pertension Society and the American Association of Medical Instruments. At present, the only device recommended for children, ac- cording to these protocols, is the QuietTrak ${ }^{37}$ Amazingly, this is a piece of auscultatory equipment, a type of device generally not adopted in pediatric studies because the noise of children in movement interferes with the accuracy of the microphone determination of the measured blood pressure value. Table 4 shows a list of some large pediatric ambulatory blood pressure monitoring studies ${ }^{36,38-42}$ and demonstrates that, as for casual blood pressure, studies are being performed without methodological homogeneity. Different devices, with different measurement protocols, cannot be considered together to generate norms.

In conclusion, as of today, the main problem for the diagnosis and management of hypertension in children is the lack of good normative data for casual and ambulatory blood pressure values. The only solution for this issue is to propose a multicenter study with a homogenous protocol, in order to obtain normal multiethnic casual and ambulatory pediatric blood pressure values. Only then will studies to correlate blood pressure level and hypertensive end-organ damage be possible.
1. Lauer RM, Clarke WR. Childhood risk factors for high adult blood pressure: the Muscatine Study. Pediatrics 1989;84(4):633-41.

2. Hoq S, Chen W, Srinivasan SR, Berenson GS. Childhood blood pressure predicts adult microalbuminuria in African Americans, but not in whites: the Bogalusa heart study. Am J Hypertens 2002;15(12):1036-41

3. Gillman MW, Ellison RC. Childhood prevention of essential hypertension. Pediatr Clin North Am 1993;40(1):179-94.

4. Kannel WB. Blood pressure as a cardiovascular risk factor: prevention and treatment. JAMA 1996;275(20):1571-6.

5. Klag MJ, Whelton PK, Randall BL. Blood pressure and endstage renal disease in men. N Engl J Med 1996;334(1):13-8.

6. Gillman MW, Cook NR. Blood pressure measurement in childhood epidemiological studies. Circulation 1995;92(4):1049-57.

7. Perloff D, Grim C, Flack J, et al. Human blood pressure determination by sphygmomanometry. Circulation 1993;88 (5 Pt 1): 2460-70.

8. Vyse TJ. Sphygmomanometer bladder length and measurements of blood pressure in children. Lancet 1987;1(8532):561-2.

9. Whincup PH, Cook DG, Shaper AG. Blood pressure measurement in children: the importance of cuff bladder size. J Hypertens 1989;7(10):845-50.

10. Arafat M, Mattoo TK. Measurement of blood pressure in children: recommendations and perceptions on cuff selection. Pediatrics 1999; 104(3): e30.

11. Prineas RJ, Jacobs D. Quality of Korotkoff sounds: bell vs diaphragm, cubital fossa vs brachial artery. Prev Med 1983;12(5):715-9.
12. Stolt M, Sjönell G, Aström H, Hansson L. Factors affecting the validity of the standard blood pressure cuff. Clin Physiol 1993;13(6):611-620.

13. Londe S. Blood pressure measurement. Pediatrics 1987;80(6):967-8.

14. Krull F, Buck T, Offner G, Brodehl J. Twenty-four hour blood pressure monitoring in healthy children. Eur J Pediatr 1993;152(7):555-8.

15. Prineas RJ, Gillum RF, Horibe H, Hannan PJ. The Minneapolis children's blood pressure study. Part 1: standards of measurement for children's blood pressure. Hypertension 1980;2(4 Pt 2):I18-24.

16. Jenner DA, English DR, Vandongen R, Beilin LJ, Armstrong BK, Dunbar D. Environmental temperature and blood pressure in 9-year-old Australian children. J Hypertens 1987;5(6):683-6.

17. Rosner B, Prineas RJ, Loggie JM, Daniels SR. Blood pressure nomograms for children and adolescents, by height, sex, and age, in the United States. J Pediatr 1993;123(6):871-86.

18. Report of the Second Task Force on Blood Pressure Control in Children - 1987. Task Force on Blood Pressure Control in Children. National Heart, Lung and Blood Institute, Bethesda, Maryland. Pediatrics 1987;79(1):1-25

19. Update on the 1987 Task Force Report on High Blood Pressure in Children and Adolescents: a working group report from the National High Blood Pressure Education Program. National High Blood Pressure Education Program Working Group on Hypertension Control in Children and Adolescents. Pediatrics 1996;98(4 Pt 1):649-58.
20. de Man SA, André JL, Bachmann H, et al. Blood pressure in childhood: pooled findings of six European studies. J Hypertens 1991;9(2):109-14.

21. Nielsen PE, Clausen LR, Olsen CA, Olsen JA. Blood pressure measurement in childhood and adolescence. International recommendations and normal limits of blood pressure. Scand J Clin Lab Invest Suppl 1989;192:7-12.

22. Beevers G, Lip GY, O'Brien E. ABC of hypertension. Blood pressure measurement. Part II - conventional sphygmomanometry: technique of auscultatory blood pressure measurement BMJ 2001;322(7293):1043-7

23. Park MK, Menard SM. Normative oscillometric blood pressure values in the first 5 years in an office setting. Am J Dis Child 1989;143(7):860-4

24. O'Brien E, Atkins N. A comparison of the British Hypertension Society and Association for the Advancement of Medical Instrumentation protocols for validating blood pressure measuring devices: can the two be reconciled? J Hypertens 1994;12(9):1089-94.

25. O'Brien E, Coats A, Owens P, et al. Use and interpretation of ambulatory blood pressure monitoring: recommendations of the British Hypertension Society. BMJ 2000;320:1128-34.

26. O'Brien E, Waeber B, Parati G, Staessen J, Myers MG. Blood pressure measuring devices: recommendations of the European Society of Hypertension. BMJ 2001;322(7285):531-6.

27. Park MK, Menard SW, Yuan C. Comparison of auscultatory and oscillometric blood pressures. Arch Pediatr Adolesc Med 2001;155(1):50-3 
28. Barker ME, Shiell AW, Law CM. Evaluation of the Dinamap 8100 and Omron M1 blood pressure monitors for use in children. Paediatr Perinat Epidemiol 2000;14(2):179-86.

29. Wells TG, Neaville WA, Arnold JR, Belsha CW. Evaluation of home blood pressure monitors in children and adolescents. Am J Med Sci 1998;315(2):110-7.

30. O’Brien E, Beevers G, Lip GY. ABC of hypertension. Blood pressure measurement. Part III - automated sphygmomanometry: ambulatory blood pressure measurement. BMJ 2001;322(7294):1110-4.

31. Lurbe E, Cremades B, Rodriguez C, Torro MI, Alvarez V, Redon J. Factors related to quality of ambulatory blood pressure monitoring in a pediatric population. Am J Hypertens 1999;12(9 Pt 1):929-33.

32. Eissa MA, Poffenbarger T, Portman RJ. Comparison of the actigraph versus patients' diary information in defining circadian time periods for analyzing ambulatory blood pressure monitoring data. Blood Press Monit 2001;6(1):21-5.

33. Belsha CW, Wells TG, McNiece KL, Seib PM, Plummer JK, Berry PL. Influence of diurnal blood pressure variations on target organ abnormalities in adolescents with mild essential hypertension. Am J Hypertens 1998;11(4 Pt 1):410-7.

34. Kapuku GK, Treiber FA, Davis HC, Harshfield GA, Cook BB, Mensah GA. Hemodynamic function at rest, during acute stress, and in the field: predictors of cardiac structure and function 2 years later in youth. Hypertension 1999;34(5):1026-31.

35. Koch VH, Colli A, Saito MI, et al. Comparison between casual blood pressure and ambulatory blood pressure monitoring parameters in healthy and hypertensive adolescents. Blood Press Monit 2000;5(5-6):281-9.

36. O'Sullivan JJ, Derrick G, Griggs P, Foxall R, Aitkin M, Wren C. Ambulatory blood pressure in schoolchildren. Arch Dis Child 1999;80(6):529-32.

37. O’Brien E, Waeber B, Parati G, Staessen J, Myers MG.Blood pressure measuring devices: recommendations of the European Society of Hypertension. BMJ 2001;322(7285):531-6.

38. Lurbe E, Redon J, Liao Y, Tacons J, Cooper RS, Alvarez V. Ambulatory blood pressure monitoring in normotensive children. J Hypertens 1994;12(12):1417-23.

39. Harshfield GA, Alpert BS, Pulliam DA, Somes GW, Wilson DK. Ambulatory blood pressure recordings in children and adolescents. Pediatrics 1994;94(2 Pt 1):180-4.

40. Lurbe E, Thijs L, Redon J et al. Diurnal blood pressure curve in children and adolescents. J Hypertens 1996;14(1):41-6

41. Reichert H, Lindinger A, Frey O, et al. Ambulatory blood pressure monitoring in healthy schoolchildren. Pediatr Nephrol 1995;9(3):282-6

42. Soergel M, Kirschstein M, Busch C, et al. Oscillometric twentyfour-hour ambulatory blood pressure values in healthy children and adolescents: a multicenter trial including 1141 subjects. J Pediatr 1997;130(2):178-84.

\section{.....Publishing information.}

Vera Hermina Koch, MD. Head of Pediatric Nephrology Unit, Instituto da Criança, Hospital das Clínicas, Universidade de São Paulo, São Paulo, Brazil.

Sources of funding: Not declared

Conflict of interest: Not declared

Date of first submission: October 28, 2002

Last received: December 2, 2002

Accepted: December 3, 2002

\section{Address for correspondence}

Vera Hermina Koch

Unidade de Nefrologia, Instituto da Criança,

Hospital das Clínicas, Universidade de São Paulo

Av. Dr. Enéas de Carvalho Aguiar, 647

São Paulo/SP - Brasil - CEP 05403-900

Tel. (+55 11) 3069-8500

Fax (+55 11) 3069-8503

E-mail:verahkk@icr.hcnet.usp.br

COPYRIGHT @ 2003, Associação Paulista de Medicina

RESUM O

Estudos epidemiológicos envolvendo medida de pressão arterial em crianças e adolescentes têm demonstrado que o valor da medida de pressão arterial na infância constituise no maior preditor dos níveis pressóricos do adulto. A hipertensão arterial no adulto eleva em duas a três vezes o risco individual de desenvolvimento de morbidade cardiovascular. O risco cardiovascular depende da pressão arterial propriamente dita, de fatores coexistentes e da presença de lesão instalada de órgãos-alvo. A acurácia na de- terminação da pressão arterial é, portanto, mandatória e um protocolo estruturado e padronizado para sua obtenção deveria ser estabelecido, possibilitando a comparação de resultados de estudos realizados em diferentes países. Este artigo discute os maiores determinantes da precisão de medida da pressão arterial casual e ambulatorial na criança.

PALAVRAS-CHAVE: Pressão. Arterial. Metodologia. Criança. Adolescente. 